\title{
Fundamental Solutions for Periodic Media
}

\author{
Sergey V. Kuznetsov \\ Institute for Problems in Mechanics, Moscow 119526, Russia \\ Correspondence should be addressed to Sergey V. Kuznetsov; kuzn-sergey@yandex.ru
}

Received 7 October 2014; Revised 27 November 2014; Accepted 1 December 2014; Published 14 December 2014

Academic Editor: Nikos Mastorakis

Copyright (C) 2014 Sergey V. Kuznetsov. This is an open access article distributed under the Creative Commons Attribution License, which permits unrestricted use, distribution, and reproduction in any medium, provided the original work is properly cited.

Necessity for the periodic fundamental solutions arises when the periodic boundary value problems should be analyzed. The latter are naturally related to problems of finding the homogenized properties of the dispersed composites, porous media, and media with uniformly distributed microcracks or dislocations. Construction of the periodic fundamental solutions is done in terms of the convergent series in harmonic polynomials. An example of the periodic fundamental solution for the anisotropic porous medium is constructed, along with the simplified lower bound estimate.

\section{Introduction}

The closest solutions in mechanics of heterogeneous media with uniformly distributed inclusions can be obtained from application of the two-scale asymptotic analysis [1-5]. In the two-scale asymptotic method, it is assumed that two fields exist: (i) the global field which is described by "slow" variables and (ii) a local field, having high oscillations; these are described by the "fast" variables; see Figure 1. Application of the two-scale asymptotic analysis to porous medium will be considered in more detail later on.

In the two-scale asymptotic method the effective elasticity tensor can be represented by

$$
\mathbf{C}_{0}=\sum_{p=1}^{N} f_{p} \mathbf{C}_{p}+\mathbf{K}, \quad \sum_{p=1}^{N} f_{p}=1
$$

where $\mathbf{C}_{0}$ is the effective elasticity tensor, $f_{p}$ is the volume fracture of the $p$ th component, $\mathbf{C}_{p}$ is the elasticity tensor of the $p$ th component, $N$ is the total number of different components of the heterogeneous material, and $\mathbf{K}$ is a correcting tensor or "corrector." It is clear from (1) that the main difficulty in determination of the effective elasticity tensor is in finding the corrector.

Remark 1. It is interesting to note that (1) covers almost all existing methods of homogenization by choosing different expressions for the corrector. (a) Thus, if $\mathbf{K}=0$ the well-known Voigt's homogenization is obtained.

(b) Taking

$$
\mathbf{K}=\sum_{p=1}^{N}-f_{p} \mathbf{C}_{p}+\left(f_{p} \mathbf{C}_{p}^{-1}\right)^{-1}
$$

and assuming that for any $p$ tensor $\mathbf{C}_{p}$ is invertible along with $\left(f_{p} \mathbf{C}_{p}^{-1}\right)$, the Reuss homogenization for the elasticity tensor comes out. Assumption that $\mathbf{C}_{p}$ is invertible for any $p$ is not valid for media with pores; in this case, the Reuss homogenization produces wrong values for the homogenized elasticity tensor.

Determination of the corrector in the two-scale asymptotic method demands the solution of the cell problem, which in turn consists of (i) a boundary value problem on the internal boundaries between inclusions and the matrix material and (ii) a periodic boundary value problem on the outer boundary of a cell. The latter one is nonclassical in the sense that it is formulated on the boundary which, due to periodicity, must have angular points and edges; see Figure 2.

Along with FEM and finite differences methods, the following other methods for obtaining a solution to the cell problem are known. The methods based on Eshelby's transformation strain were applied to analysis of isotropic medium with ellipsoidal inclusions [6-8]. The advantage 


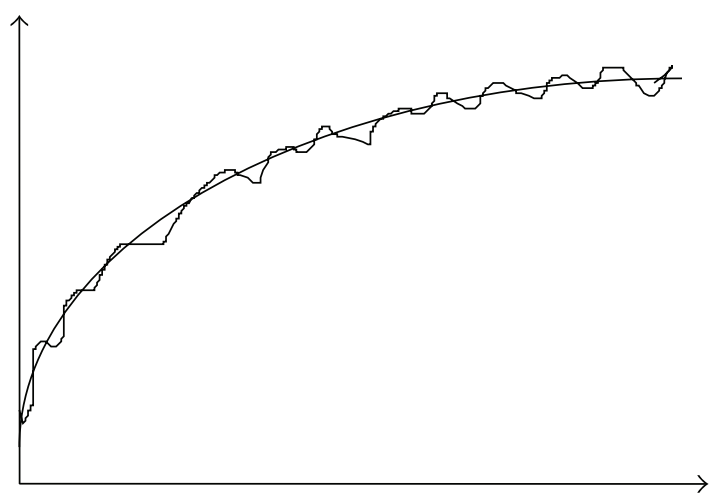

FIGURE 1: "Slow" and "fast" varying processes (horizontal axis can refer to either time or distance).

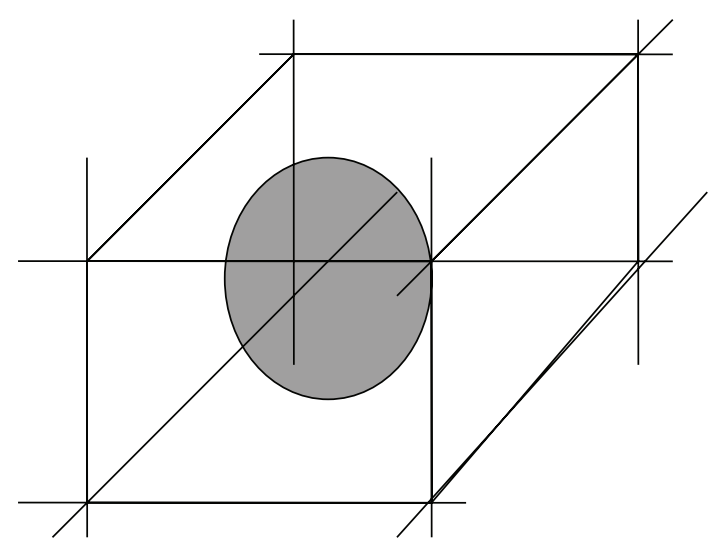

FIGURE 2: The typical periodic cell.

of these methods resides in their principle possibility to analyze media with anisotropic components, while from the computational point of view these are not very convenient since they lead to the three-dimensional integral equations with weakly singular kernels.

The media with isotropic components were studied by a method based on the periodic fundamental solution for isotropic medium $[9,10]$; such a fundamental solution was originally constructed in [11]. Because of multipolar expansions used for the solution of the inner boundary value problem, this method is confined to inclusions of spherical form. A similar approach was also used in the case of isotropic composites, but it was based on the Galerkin technique for solution of the inner boundary value problem [12].

Presumably, for the first time periodic fundamental solutions for media with arbitrary anisotropy were developed in [13]. In combination with the boundary integral equation method (BIEM) these fundamental solutions were applied to the cell problem for composites with anisotropic inhomogeneities and porous media in $[14,15]$, while analysis of microstructural stresses in the matrix material was considered in [16]. Problems of wave scattering by pores were studied in $[17,18]$ by the same method. Some of obvious advantages of this method are due to their potential possibility to reduce the solution of the inner boundary value problem to a summation of rapidly convergent series, while periodic boundary conditions on the outer boundary are satisfied automatically due to periodicity of the fundamental solution.

The following analyses cover both construction and properties of the periodic fundamental solutions and application of these solutions to analysis of porous anisotropic media with uniformly distributed pores.

\section{Basic Notations}

Initially a homogeneous elastic anisotropic medium is considered. The equations of equilibrium can be written in the following form:

$$
\mathbf{A}\left(\partial_{x}\right) \mathbf{u}=-\operatorname{div}_{x} \mathbf{C}_{0} \cdots \nabla_{x} \mathbf{u}=0,
$$

where $\mathbf{u}$ is a displacement field. It is assumed that the tensor of elasticity satisfies the condition of positive definiteness, which is generally adopted for problems of mechanics of inhomogeneous media.

Applying the Fourier transform

$$
f^{\wedge}(\xi)=\int f(\mathbf{x}) \exp (2 \pi i \mathbf{x} \cdot \xi) d x, \quad \xi \in R^{3},
$$

to (3) gives the following symbol of the operator A:

$$
\mathbf{A}^{\wedge}(\xi)=(2 \pi)^{2} \xi \cdot \mathbf{C}_{0} \cdot \xi .
$$

From the definition of the fundamental solution $\mathbf{E}$ of (3) the following formula for the corresponding symbol can be written:

$$
\mathbf{E}^{\wedge}(\xi)=\mathbf{A}^{\wedge}(\xi)^{-1}
$$

Formula (6) shows that symbol $\mathbf{E}^{\wedge}$ is also strongly elliptic, positively homogeneous of degree -2 with respect to $|\xi|$, and analytical everywhere in $R^{3} \backslash 0$.

Remark 2. The Fourier inversion of the expression (6) and procedures for construction of the fundamental solution are discussed in [19-21].

\section{Periodic Fundamental Solution}

Consider a homogeneous anisotropic medium, loaded by the periodically distributed force singularities, located in nodes m of a spatial lattice $\Lambda$, Figure 3 .

Let $\mathbf{a}_{i}(i=1,2,3)$ be linearly independent vectors of the main periods of the lattice, so that each of the nodes can be represented in the following form:

$$
\mathbf{m}=\sum_{i} m_{i} \mathbf{a}_{i}
$$

where $m_{i} \in Z$ are integer-valued coordinates of the node $\mathbf{m}$ in the basis $\left(\mathbf{a}_{i}\right)$. The adjoint basis $\left(\mathbf{a}_{i}^{*}\right)$ is introduced in such a manner that $\mathbf{a}_{i}^{*} \cdot \mathbf{m}=m_{i}$. The lattice corresponding to the adjoint basis is denoted by $\Lambda^{*}$. 


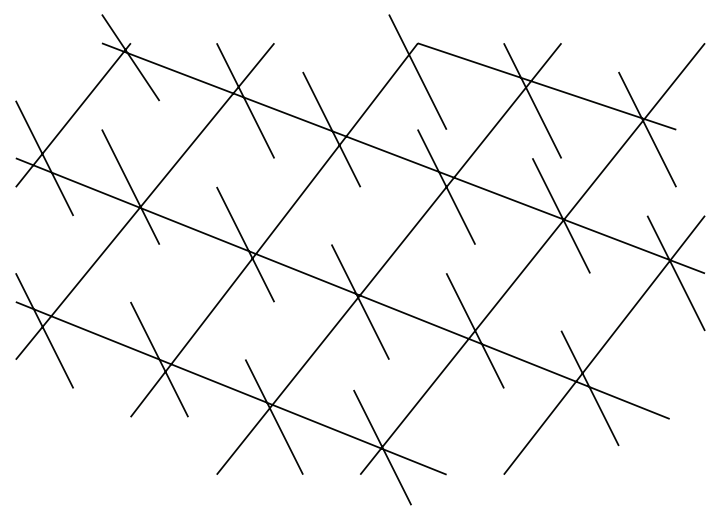

FIgure 3: Periodic lattice.

Now, periodic delta-function corresponding to singularities disposed in the nodes of the lattice $\Lambda$ has the following form:

$$
\delta_{p}(\mathbf{x})=V_{\mathrm{Q}}^{-1} \sum_{\mathbf{m}^{*} \in \Lambda^{*}} \exp \left(-2 \pi i \mathbf{x} \cdot \mathbf{m}^{*}\right)
$$

where $V_{Q}$ is the volume of the fundamental region (cell) $Q$. Formula (8) defines a periodic delta-function uniquely.

Substitution of the periodic fundamental solution $\mathbf{E}_{p}$ in (3) should produce

$$
\mathbf{A}\left(\partial_{\mathbf{x}}\right) \mathbf{E}_{p}(\mathbf{x})=\delta_{p}(\mathbf{x}) \mathbf{I},
$$

where $\mathbf{I}$ is the identity matrix. Looking for $\mathbf{E}_{p}$ also in the form of harmonic series and taking into account representation (8), it is possible to obtain

$$
\mathbf{E}_{p}(\mathbf{x})=V_{\mathrm{Q}}^{-1} \sum_{\mathbf{m}^{*} \in \Lambda_{0}^{*}} \mathbf{E}^{\wedge}\left(\mathbf{m}^{*}\right) \exp \left(-2 \pi i \mathbf{x} \cdot \mathbf{m}^{*}\right),
$$

where $\Lambda_{0}^{*}$ is an adjoint lattice without the zero node. It should be noted that formula (10) defines a periodic fundamental solution up to an additive (tensorial) constant.

Lemma 3 (see [14]). The series on the right side of (10) is convergent in the $L^{1}$-topology, defining fundamental solution of the class $\overline{L^{1}}\left(Q, R^{3} \otimes R^{3}\right)$, where $\overline{L^{1}}$ is a class of integrable in $Q$ functions with the zero mean value.

\section{Effective Elasticity Tensor}

For clarity and simplicity it will be assumed further that the considered medium has only one kind of uniformly distributed inhomogeneities placed in nodes of spatial lattice $\Lambda$ (refer to Figure 3 ). The region occupied by an individual inhomogeneity in a cell $Q$ is denoted by $\Omega$.

Two-scale asymptotic analyses being applied to such a medium produce the following expression for the corrector [14]:

$$
\mathbf{K}=-V_{\mathrm{Q}}^{-1} \int_{\partial \Omega} \mathbf{C} \cdots\left(\nu_{\mathbf{Y}} \otimes \mathbf{H}(\mathbf{Y})\right) d Y
$$

where $\mathbf{Y}$ are "fast" variables and $\mathbf{H}$ is the third-order tensorial field, being a solution of the following boundary value problem:

$$
\begin{gathered}
\mathbf{A}\left(\partial_{\mathbf{Y}}\right) \mathbf{H}(\mathbf{Y})=0, \quad \mathbf{Y} \in Q \backslash \Omega, \\
\left.\mathbf{T}\left(\nu_{\mathbf{Y}}, \partial_{\mathbf{Y}}\right) \mathbf{H}(\mathbf{Y})\right|_{\partial \Omega}=-\nu_{\mathbf{Y}} \cdot \mathbf{C} .
\end{gathered}
$$

In (11) and (12) $\nu_{\mathrm{Y}}$ represents the field of external unit normal to the boundary $\partial \Omega$, and the elasticity tensor $\mathbf{C}$ is defined by

$$
\mathbf{C}=\mathbf{C}_{2}-\mathbf{C}_{1} \text {, }
$$

where $\mathbf{C}_{2}$ is referred to the matrix material and $\mathbf{C}_{1}$ to inclusions. Strong ellipticity of the tensor $\mathbf{C}$ is also assumed. It should be noted that in (13) tensor $\mathbf{C}_{1}$ can vanish in the case of voids.

Lemma 4 (see [14]). Under assumptions stated above, the boundary value problem (12) admits the unique solution.

Solution of the boundary value problem (12) is constructed by the boundary integral equation method, giving the following representation for the desired solution [14]:

$$
\left(\frac{1}{2} \mathbf{I}+\mathbf{S}\right) \mathbf{H}\left(\mathbf{Y}^{\prime}\right)=\mathbf{H}_{c} \quad \mathbf{Y}^{\prime} \in \partial \Omega
$$

where $\mathbf{H}_{c}$ is a constant tensor and $\mathbf{S}$ is a singular integral operator resulting from a restriction of the double-layer potential on the surface $\partial \Omega$. Some of the relevant properties of operator $\mathbf{S}$ are discussed in [15].

Substitution of (10) for periodic fundamental solutions in the expression for the operator $S$ allows obtaining a lower (in energy) bound for the corrector; that is,

$$
\mathbf{K}_{l}=-8 \pi^{2} V_{\mathrm{Q}}^{-2} \sum_{\mathbf{m} * \in \Lambda_{0}^{*}}\left|\chi_{\Omega}^{\wedge}\right|^{2} \mathbf{C} \cdot \mathbf{m}^{*} \otimes \mathbf{E}^{\wedge}\left(\mathbf{m}^{*}\right) \otimes \mathbf{m}^{*} \cdots \mathbf{C}
$$

where $\chi_{\Omega}^{\wedge}$ is the Fourier image of the characteristic function of the region $\Omega$. An expression for the upper bound can be obtained similarly $[14,15]$.

Theorem 5 (see $[14,15]$ ). Series appearing on the right side of (15) is absolutely convergent, provided $\Omega$ is a proper open region in $\mathrm{Q}$.

Remark 6. Proof of convergence for very thin inclusions, including cracks, is to be studied separately, as the special asymptotic analysis is needed.

\section{Microstructural Stresses and Scattering Cross-Sections}

As was shown in $[15,16]$, the energy level $W_{\text {osc }}$ of microstructural highly oscillating stresses for porous medium is defined by

$$
W_{\mathrm{osc}}=\frac{1}{2} \varepsilon_{0} \cdot \mathbf{K} \cdot \varepsilon_{0},
$$


where $\varepsilon_{0}$ represents the uniform deformation field and $\mathbf{K}$ is the corrector.

Similarly, having applied terminology used in quantum mechanics, scattering cross-section $S$ for the porous medium has the following form:

$$
S=(1-f)^{-1}\left|\frac{\varepsilon_{0} \cdot \mathbf{K} \cdot \varepsilon_{0}}{\varepsilon_{0} \cdot \mathbf{C} \cdot \varepsilon_{0}}\right|,
$$

where $f$ is the porous ratio and $\mathbf{C}=\mathbf{C}_{2}$ is the elasticity tensor of the matrix.

Remark 7. Problems for obtaining expressions for microstructural stresses and scattering cross-sections related to media with inclusions of nonzero stiffness are not studied yet by the two-scale asymptotic analysis and periodic fundamental solutions.

\section{Conclusions}

The developed methodology allowed us to construct the spatially periodic fundamental solution (10) for periodic media with anisotropic components. On the basis of thus constructed periodic fundamental solution, the closed form expression for the corrector tensor for porous medium with anisotropic matrix was obtained (11), along with the approximate formula (15).

An estimate for the scattering cross-section for porous medium with anisotropic matrix was derived; see (17).

\section{Conflict of Interests}

The author declares that there is no conflict of interests regarding the publication of this paper.

\section{References}

[1] N. S. Bakhvalov, "Homogenized characteristics of bodies with periodic structure," Doklady Akademii Nauk SSSR, vol. 218, pp. 1046-1048, 1974, (Russian).

[2] A. Bensoussan, J. L. Lions, and G. Papanicolaou, Asymptotic Analysis for Periodic Structures, North-Holland, Amsterdam, The Netherlands, 1978.

[3] E. Sanchez-Palencia, "Homogenization method for the study of composite media," in Asymptotic Analysis II, pp. 192-214, 1983.

[4] G. Allaire, "Homogenization and two-scale convergence," SIAM Journal on Mathematical Analysis (SIMA), vol. 23, no. 6, pp. 1482-1518, 1992.

[5] J. Diaz-Alban and N. Masmoudi, "Asymptotic analysis of acoustic waves in a porous medium: initial layers in time," Communications in Mathematical Sciences, vol. 10, no. 1, pp. 239-265, 2012.

[6] S. Nemat-Nasser, T. Iwakuma, and M. Hejazi, "On composites with periodic structure," Mechanics of Materials, vol. 1, no. 3, pp. 239-267, 1982.

[7] S. Nemat-Nasser and M. Taya, "On effective moduli of an elastic body containing periodically distributed voids," Quarterly of Applied Mathematics, vol. 39, no. 1, pp. 43-59, 1981.

[8] S. Nemat-Nasser and M. Taya, "On effective moduli of an elastic body containing periodically distributed voids: comments and corrections," Quarterly of Applied Mathematics, vol. 43, pp. 187188,1985

[9] A. S. Sangani and A. Acrivos, "Slow flow through a periodic array of spheres," International Journal of Multiphase Flow, vol. 8, no. 4, pp. 343-360, 1982.

[10] A. S. Sangani and W. Lu, "Elastic coefficients of composites containing spherical inclusions in a periodic array," Journal of the Mechanics and Physics of Solids, vol. 35, no. 1, pp. 1-21, 1987.

[11] H. Hasimoto, "On the periodic fundamental solutions of the Stokes equations and their application to viscous flow past a cubic array of spheres," Journal of Fluid Mechanics, vol. 5, pp. 317-328, 1959.

[12] K. C. Nunan and J. B. Keller, "Effective elasticity tensor of a periodic composite," Journal of the Mechanics and Physics of Solids, vol. 32, no. 4, pp. 259-280, 1984.

[13] S. V. Kuznetsov, "Periodic fundamental solutions for anisotropic media," Izvestiya RAN. Mekhanika Tverdogo Tela, no. 4, pp. 99104, 1991 (Russian).

[14] S. V. Kuznetsov, "The effective elasticity tensors for dispersed composites," Journal of Applied Mathematics and Mechanics, vol. 57, no. 1, pp. 117-124, 1993.

[15] S. V. Kuznetsov, "Porous media with internal pressure," Izvestia RAN. Mekhanika Tverdogo Tela, no. 6, pp. 22-28, 1993 (Russian).

[16] S. V. Kuznetsov, "Microstructural stresses in porous media," Prikladnaya Mekhanika, vol. 27, no. 8, pp. 23-28, 1991.

[17] S. V. Kuznetsov, "Wave scattering in porous media," Izvestiya RAN. Mekhanika Tverdogo Tela, no. 3, pp. 81-86, 1995 (Russian).

[18] S. V. Kuznetsov, "Scattering of elastic waves in porous media," International Journal of Latest Research in Science and Technology, vol. 4, pp. 61-64, 2014.

[19] S. V. Kuznetsov, "Some singular solutions of the theory of elasticity," Journal of Applied Mathematics and Mechanics, vol. 60 , no. 5, pp. 863-865, 1996.

[20] S. V. Kuznetzov, "Direct boundary integral equation method in the theory of elasticity," Quarterly of Applied Mathematics, vol. 53, no. 1, pp. 1-8, 1995.

[21] S. V. Kuznetsov, "On the operator of the theory of cracks," Comptes Rendus de l'Académie des Sciences, vol. 323, pp. 427432, 1996. 


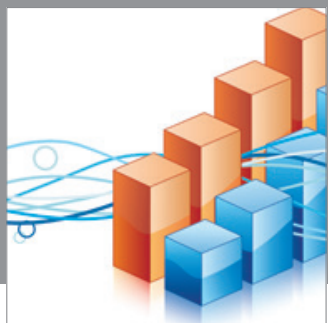

Advances in

Operations Research

mansans

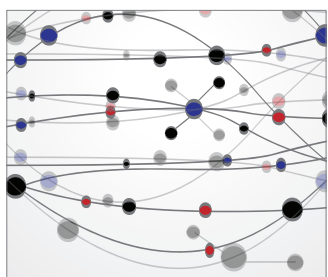

The Scientific World Journal
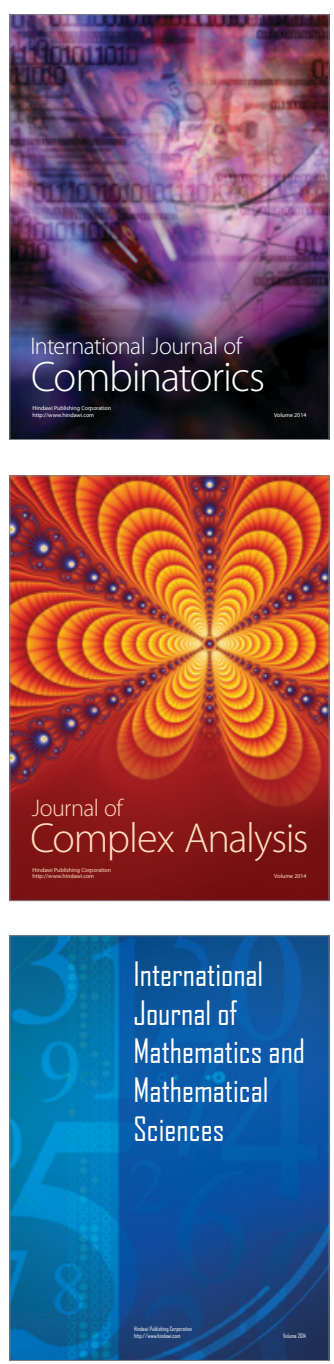
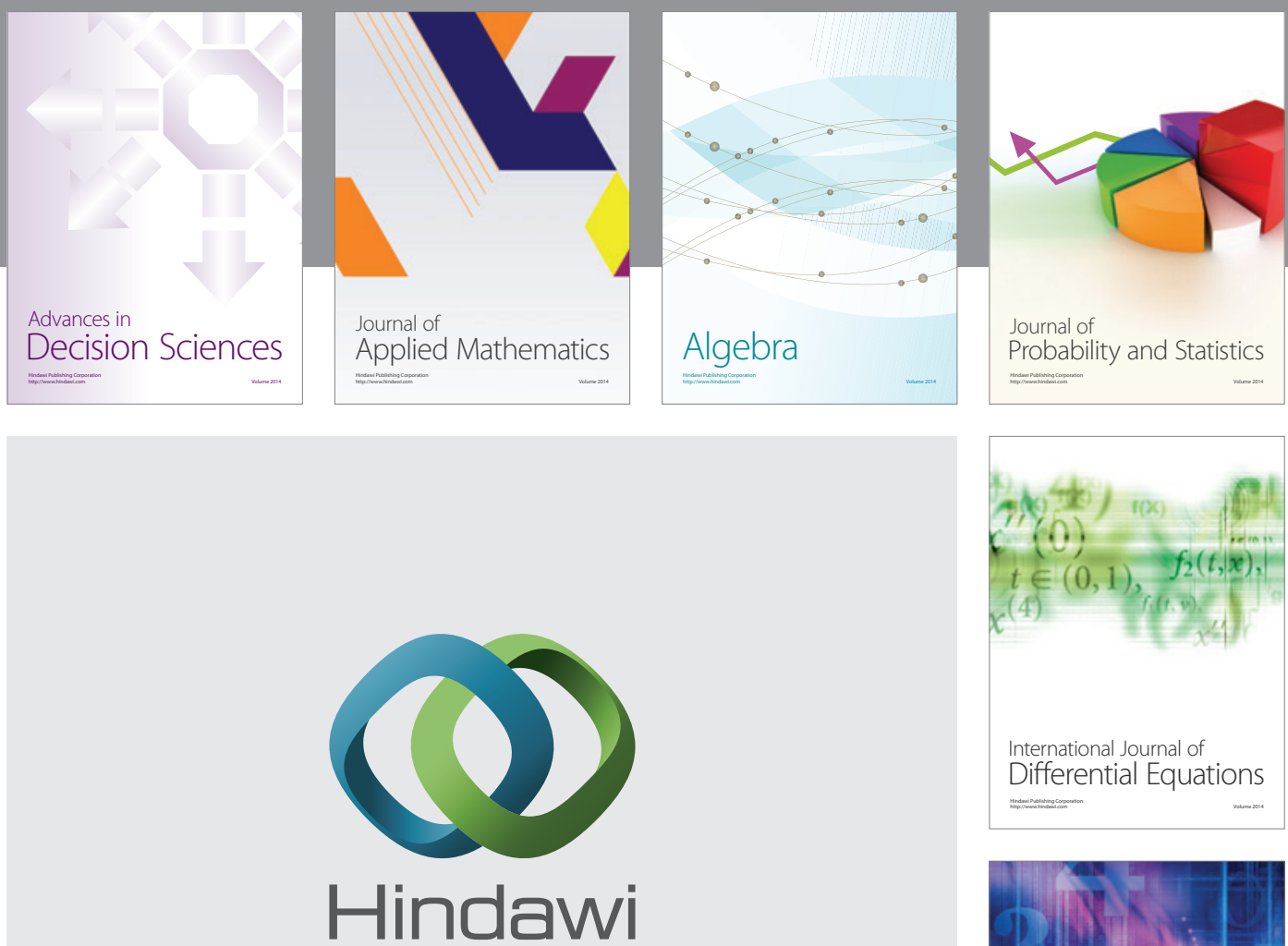

Submit your manuscripts at http://www.hindawi.com
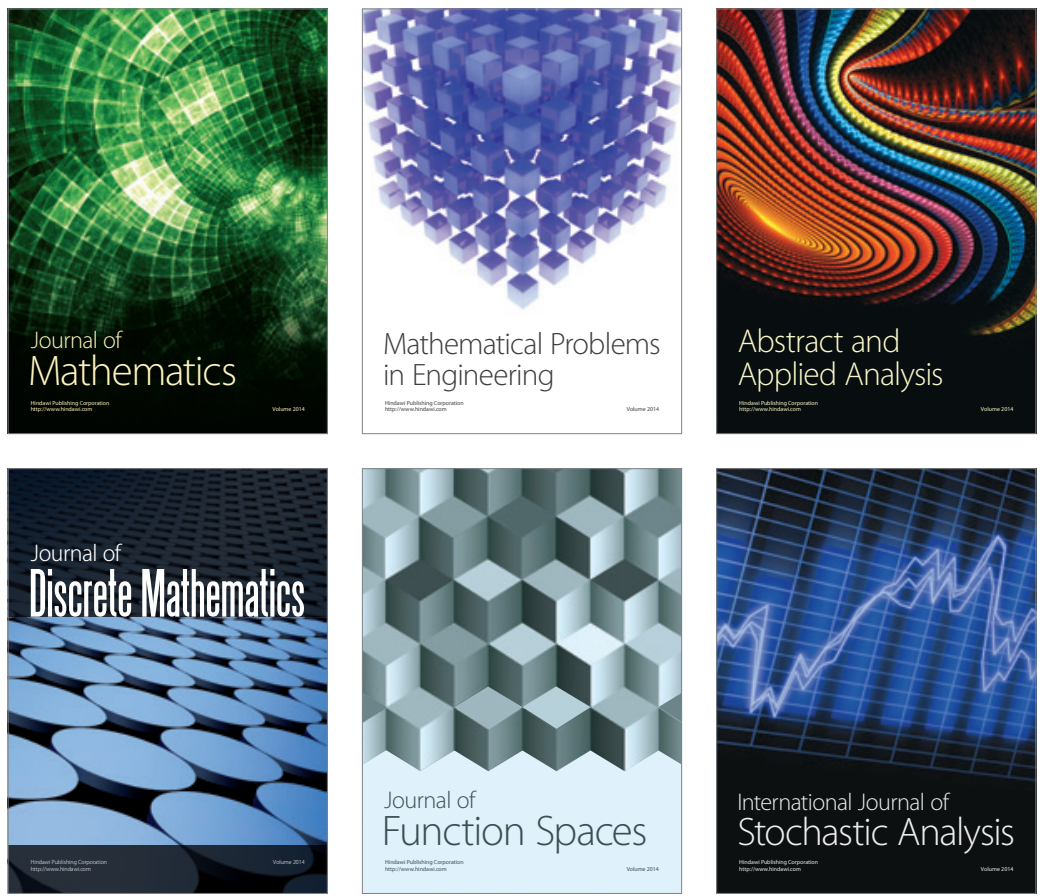

Journal of

Function Spaces

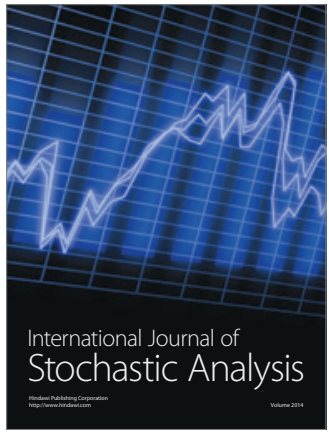

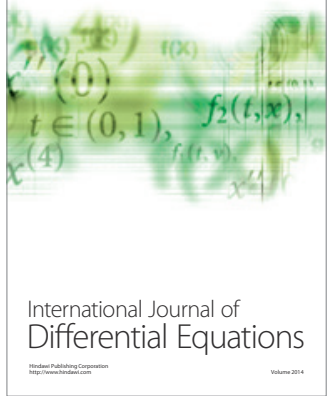
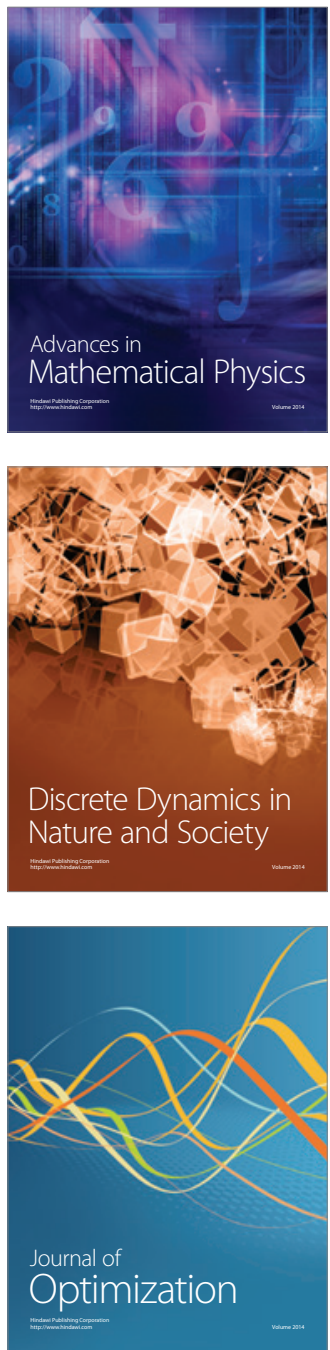